\title{
¿ESTÁ JUSTIFICADA LA MALA IMAGEN DE LA ADMINISTRACIÓN DE JUSTICIA ESPAÑOLA? ¿ES UN PROBLEMA DE INVERSIÓN?: UNA COMPARATIVA EUROPEA MEDIANTE EL ANÁLISIS DEA
}

\section{IS THE SPANISH JUDICIAL ADMINISTRATION'S POOR IMAGE JUSTIFIED? IS IT A PROBLEM OF INVESTMENT? A EUROPEAN COMPARISON USING DEA ANALYSIS}

Vázquez Cueto, María José (Universidad de Sevilla) *

Gutiérrez López, Francisco (Audiencia Provincial de Sevilla) ${ }^{* *}$

\section{RESUMEN}

La justicia es un bien público y, en consecuencia, en el seno de la Administración Pública, el correcto funcionamiento de la Administración de Justicia tiene capital importancia para una sociedad. En la actualidad, el sistema judicial español es objeto de severas críticas, que lo califican de ineficiente y tardío. Un gran sector social y profesional de la Administración de Justicia considera que la falta de medios es la causa de su mal funcionamiento, y reclaman mayor inversión. En este trabajo analizamos si ambas aseveraciones son acertadas: si es ineficiente la Administración de Justicia española y si un mayor gasto conllevaría a la mejora de este servicio público. Aplicando la metodología de Análisis Envolvente de Datos (DEA), determinamos la eficiencia de la Administración de Justicia española en relación con otras Administraciones de Justicia europeas y, mediante el análisis tobit, relacionamos las puntuaciones DEA y el gasto público. Nuestros resultados concluyen que un mayor esfuerzo en el gasto conllevará a una mejor posición.

Palabras claves: Administración de Justicia, Eficiencia, DEA, Tobit, Europa. JEL: H11, H57, K0.

\footnotetext{
ABSTRACT

Justice is a public good. Therefore, within the core of the Public Administration, the proper functioning of the Judicial Administration is of utmost importance to society. Currently, the Spanish Judicial System is being subject to sharp criticism that qualifies it as inefficient and belated. A large social and professional sector of the Judicial Administration considers that the lack of means is the reason behind its poor functioning and so, they demand greater investment. Throughout this paper the accuracy of both assertions will be analysed: whether the Spanish Judicial Administration is inefficient and whether a greater expenditure would lead to the improvement of such public service. By applying the Data Envelopment Analysis (DEA) methodology, the efficiency of the Spanish Judicial Administration is determined and

* Departamento de Economía Aplicada III, Área Métodos cuantitativos para la Economía y Empresa, Facultad de Ciencias Económicas y Empresariales, Avda. Ramón y Cajal, 1, 41018, Sevilla. pepi@us.es

** Audiencia Provincial de Sevilla, CP 41071. gutivalca@gmail.com

Recibido: Abril de 2017. Aceptado: Octubre de 2017.
} 
compared to that of other European Judicial Administrations. Through the Tobit analysis, the DEA levels are related to the public spending. Our outcomes show that a greater expenditure effort will result into a better ranking position.

Key words: Justice Administration, Efficiency, DEA, Tobit, Europe. JEL: H11, H57, K0.

\section{INTRODUCCIÓN}

Según Santos Pastor Prieto ${ }^{1}$, destacado estudioso y precursor en nuestro país del análisis de lo que se denomina la Economía de la Justicia, "El sistema jurídico es un componente fundamental para la vida de las personas. También para el desempeño económico. Las economías de mercado necesitan sistemas jurídicos que incentiven la creación de valor mediante adecuados derechos de propiedad, estimulen los contratos dotándolas de un derecho de contratos que reduzca los costes de transacción y garanticen y promuevan el desarrollo de los derechos y libertades. La justicia es parte del sistema jurídico y su función principal consiste en hacer que las normas no sean papel mojado. Las leyes que no se aplican, por buenas que sean, no surten efectos sociales. Cuando la justicia es lenta, costosa $o$ ineficaz, las personas sufren injustas $e$ ineficientes violaciones en sus derechos $y$ libertades".

La importancia que la justicia tiene en la sociedad también es visible por las consecuencias económicas que su mal funcionamiento acarrea, como ya señalaron Ballbé y Padrós $^{2}$ (1997) y Santos Pastor ${ }^{3}(2003)^{4}$. En este mismo sentido se pronuncian los trabajos de Nieto (2005), Iglesias y Arias (2006), Padilla, et al. (2007), Ménard y Marais (2008), Palumbo et al. (2013), Hayo y Voigt (2014), Ippoliti et al. (2015) y, para el caso español, Fabbri (2010), García Posada y Mora Sanguinetti (2013), Jimeno et al. (2015) y, más recientemente, Mora Sanguinetti et al. (2016) ${ }^{5}$, entre otros.

Sin embargo, y tal como señala implícitamente el trabajo de Voigt y El Bialy (2012), la Administración de Justicia no solo debe funcionar bien sino que además debe parecerlo. En la actualidad, el sistema judicial español no pasa por sus mayores cotas de popularidad, aumentando el número de personas que la consideran "el principal problema que existe actualmente en España” (barómetros del Centro de Investigaciones Sociológicas (CIS) correspondientes a 2015 y 2016 ${ }^{6}$ ). Junto a ello, existe un numeroso sector social y profesional de la propia Administración de Justicia que considera que la falta de medios es la causa de su mal funcionamiento y reclaman mayor inversión.

Bajo estas premisas planteamos este trabajo, que tiene como principal objetivo poner en valor el funcionamiento de la Administración de Justicia en España analizando en primer lugar su eficiencia en comparación con otros países europeos, para, en una segunda etapa, determinar, si es posible, cuáles son las razones que podrían explicar su posicionamiento

\footnotetext{
${ }^{1}$ Pastor y Rosales (2005, p. 103).

2 Ballbé y Padrós (1997, p. 49).

${ }^{3}$ Pastor (2003, p. 105).

${ }^{4}$ Ver Círculo de Empresarios (2003).

${ }^{5}$ Puede profundizarse sobre este hecho en Mora-Sanguinetti (2016).

${ }^{6}$ Los barómetros del Centro de Investigaciones Sociológicas (CIS) son las encuestas de opinión que, con periodicidad mensual, se realizan para hacer el seguimiento de las opiniones y actitudes de la sociedad española ante distintas situaciones o acontecimientos de la actualidad.
} 
relativo, prestando especial atención a la relación con el esfuerzo de gasto realizado, pudiendo determinar, de este modo, si el problema es de falta de inversión, o no.

Para alcanzar los anteriores objetivos es necesario conocer y relacionar los datos oficiales relativos a la inversión, medios personales y funcionamiento del sistema judicial, porque la información resulta esencial para realizar un análisis científico que conlleve a una planificación adecuada.

Así, tras esta introducción, este trabajo desarrolla los siguientes epígrafes: en primer lugar expondremos la metodología a seguir en el análisis y una breve revisión bibliográfica de la aplicación de la misma; en el epígrafe tres se presentan las variables que se utilizarán para determinar la eficiencia, así como sus descriptivos. En el cuarto epígrafe se analizan los resultados obtenidos con el método elegido y se determinan, en el epígrafe cinco, las relaciones de estos con las variables utilizadas. En base a ello se exponen, en el epígrafe seis, las conclusiones.

\section{METODOLOGÍA}

En su versión más simple e intuitiva, una unidad productiva es eficiente cuando es capaz de producir los máximos outputs con los mínimos inputs posibles, sujeto a las restricciones de entorno operativo apropiadas (Sufijan, 2011; Coelli et al., 2005; Banker et al., 1984).

En el ámbito económico, la eficiencia se define como una medida que pone en relación los medios empleados con los fines obtenidos, considerándose una determinada técnica, procedimiento o sistema de producción eficiente cuando, para un determinado nivel de inputs, es capaz de producir la máxima cantidad de output de entre todas las técnicas, procedimientos o sistemas de producción considerados, o si para alcanzar un determinado output emplea la menor cantidad de inputs de entre todas las técnicas, procedimientos o sistemas de producción considerados (Lowell, 1993).

De la propia definición se deduce que el concepto de eficiencia es relativo y exige de la comparación de la unidad productiva objeto de análisis con otras con las que sus outputs e inputs mantengan relación. Así, por ejemplo, y midiendo en unidades monetarias, si tenemos tres unidades A, B y C que utilizan dos inputs para producir un output según los datos del cuadro 1.

\begin{tabular}{|c|c|c|c|}
\hline \multicolumn{5}{|c|}{ CUADRO 1. EJEMPLO ILUSTRATIVO } \\
\begin{tabular}{|c|c|c|c|}
\hline Unidad productiva & Output & Input 1 & Input 2 \\
\hline A & 12 & 6 & 12 \\
\hline$B$ & 4 & 8 & 6 \\
\hline$C$ & 5 & 3 & 10 \\
\hline
\end{tabular} \\
Fuente: Elaboración propia.
\end{tabular}

A es más eficiente que $C$ puesto que, usando el doble de la cantidad de los inputs 1 y 2 que usa C, consigue un output mayor al doble que C: 12 de A frente a 5 de C. Esta comparación, que ha resultado inmediata para A y C, para B obligaría a plantear un

sistema de ecuaciones de la siguiente forma:

$$
\begin{aligned}
& \operatorname{input1}(B)=\frac{8}{12} * \operatorname{input1}(A)+\frac{8}{6} * \operatorname{input1}(C) \\
& \text { input2(B) }=\frac{8}{12} * \operatorname{input2}(A)+\frac{8}{6} * \operatorname{input2}(C)
\end{aligned}
$$


Luego, de la experiencia, el output de B debería ser

otuput $(B)=\frac{8}{12} *$ output $(A)+\frac{8}{6} * \operatorname{output}(C)=\frac{80}{12}+\frac{40}{6}=\frac{80}{6}$

y sin embargo es 4, es decir B resultaría ineficiente, en concreto su "eficiencia comparativa" sería $4 /(40 / 3)=0.3333$, cociente entre el output conseguido y el que potencialmente debería haber tenido. ${ }^{7}$

El problema, obviamente, se hace más complejo a medida que aumentan las unidades productivas a comparar y el número de inputs y outputs considerados.

Para resolverlo Charnes et al. (1978), basándose en el trabajo de Farrell (1957), proponen la metodología del Análisis Envolvente de Datos, en adelante DEA.

El método utiliza un procedimiento de programación lineal para identificar empíricamente, a través de los datos proporcionados, una función de producción, de aquí que se encuadre dentro de los métodos de estimación no paramétrica, y compara todas las unidades similares teniendo en cuenta simultáneamente todos los inputs y outputs. El modelo desarrollado por los autores mencionados considera rendimientos a escala constantes y es conocido por las siglas de sus autores CCR, en contraposición a la extensión posterior a modelos con rendimientos a escala variables, desarrollado por Banker et al. (1984), al que se le conoce como modelo DEA BCC.

La flexibilidad de los modelos, al no necesitar conocer a priori la función de producción, además de permitir variables medidas en distintas unidades, lo hacen idóneos para ser aplicado en varios campos de conocimiento, tanto en su versión CCR como en BCC ${ }^{8}$.

De hecho, alcanzan inmediatamente altas cotas de popularidad, aplicados en el análisis de eficiencia en sectores tales como la energía (Schuschny, 2007), la industria (Coelli et al., 2005), la banca (Palečková, 2015), los hospitales (Ley, 1991), la educación (Veiderpass y McKelvey, 2014; Nazarko y Šaparauskas, 2014; El-Mahgary et al., 2014). Los trabajos de Liu et al. (2013 a y b) nos muestran en qué áreas científicas se ha aplicado en mayor medida esta metodología. Y, aunque en el sector público que nos ocupa y para el área geográfica que consideramos no abunden trabajos, sí que podemos citar algunos determinantes.

Así, para España, es precursor el trabajo de Pedraja y Salinas (1995), que analiza la eficiencia con la que actúan en 1991 los Tribunales Superiores de Justicia en España en las Jurisdicción Contenciosa-Administrativa: aplicando el análisis DEA concluyen que solo cinco de las veintiuna sedes analizadas son eficientes, enfatizando la necesidad de disponer de datos más desagregados para realizar análisis con mayor profundidad. Más recientemente García Rubio y Rosales López (2010), evalúan la eficiencia judicial en Andalucía, con datos de 65 de los 115 Juzgados de Primera Instancia existentes en la Comunidad en 2008, resultando que solo 11 de ellos son eficientes ${ }^{9}$.

De igual forma las cortes noruegas fueron objeto de análisis mediante este método en 1992 por Kittelsen y Førsund; y, aunque restringido a los jueces de paz, en Bélgica por

\footnotetext{
${ }^{7}$ Para otros ejemplos ilustrativos ver Mar y Woracker (1996).

${ }^{8}$ Los desarrollos matemáticos de ambos modelos, tanto de los que están orientados a la maximización del output, como de los que lo están a la minimización del input, pueden consultarse en los trabajos originales de los autores señalados.

9 Este trabajo completa el realizado por Rosales López (2008) sobre la misma región y aplicando otra metodología.
} 
Tulkens (1993). Más recientes son los trabajos sobre las cortes italianas, Finocchiaro y Guccio (2014) y Peyrache y Zago (2016), y las portuguesas, Santos y Amado (2014). Sin embargo, de los 46 estudios que recoge Voigt (2016), solo uno de ellos realiza un análisis comparativo entre países ${ }^{10}$. Creemos, por tanto, que nuestro trabajo viene a paliar este hueco en la investigación, realizando un análisis comparativo de la eficiencia en las administraciones de justicia de 34 países de la OCDE con el objetivo de situar a la Administración de Justicia española en el contexto europeo y, posteriormente, analizar hasta qué punto un mayor gasto en Justicia garantiza mejores resultados.

Para la obtención de la puntuación de eficiencia técnica, una vez seleccionados los inputs y outputs, y considerando que estamos ante unidades intensivas de producción, utilizaremos el modelo CCR orientado a la maximización del output, y posteriormente relajaremos la suposición de rendimientos constantes a escala y aplicaremos el modelo BCC, lo que nos permitirá detectar las ineficiencias de escala.

Las puntuaciones obtenidas se relacionarán mediante la regresión Tobit con el gasto realizado por cada país en su Administración de Justicia, medido con tres indicadores distintos: porcentaje de gasto en administración de justicia con respecto al gasto del estado, gasto en administración de justicia por habitante medido en euros, y número de jueces y fiscales por cada 100.000 habitantes.

\section{DATOS UTILIZADOS EN EL ESTUDIO}

En septiembre de 2002 se creó en el seno del Consejo de Europa la "European Commission for the Efficiency of Justice (CEPEJ)". Con ello se perseguía "presentar una fotografía lo más precisa posible de los sistemas judiciales de los Estados europeos, para poder compararlos". Su misión era recopilar datos relacionados con los sistemas judiciales de los países integrantes para mejorar su funcionamiento. Se quiso, con ello, establecer "una estructura capaz de proponer soluciones concretas para mejorar la equidad, calidad y eficacia de la justicia en Europa, para reforzar la confianza de los justiciables en su sistema nacional y limitar los recursos ante la Corte de Estrasburgo, motivados por las disfunciones de la justicia, contrarios al artículo 6 de la Convención Europea de los Derechos del Hombre" 11 .

Para conseguir estos objetivos se elabora un cuestionario destinado a recolectar en los Estados miembros la información cualitativa y cuantitativa sobre el funcionamiento cotidiano de los sistemas judiciales. El objetivo principal era asegurar la recolección de datos homogéneos de un ejercicio al otro, permitiendo así las comparaciones de datos en el tiempo, a partir de la determinación y análisis de las primeras series estadísticas.

Desde que en 2004 se confeccionó el informe piloto referido a datos del año 2002, cada dos años la CEPEJ publica un nuevo informe con los datos que extrae de las encuestas que envía a todos los países, en las que, entre otras, se formulan preguntas sobre los gastos públicos, los medios materiales y humanos al servicio de la justicia y los resultados de la actividad jurisdiccional.

\footnotetext{
10 En este sentido, aunque como resultado intermedio, podemos citar también el trabajo de Deyneli (2012) que relaciona la eficiencia de la justicia con los incentivos o salarios a los jueces mediante un análisis DEA en dos etapas, en la primera de las cuales establece un ranking de varios países en función de dicha eficiencia.

11 Resolución Res (2002)12 del Comité de Ministros del Consejo de Europa donde se crea la European Commission for the Efficiency of Justice (CEPEJ).
} 
Es indudable que la creación de la CEPEJ supuso un gran avance y una herramienta muy eficaz para unificar y mejorar el funcionamiento de la administración de justicia en Europa a través, precisamente, de la información, pero los datos deben tomarse con cierta prevención porque resulta ciertamente complejo comparar sistemas judiciales diversos en sus estructuras, competencias y organización. En este sentido, con datos referidos a 2012, últimos publicados por la CEPEJ en el momento de la realización de este estudio, y basándonos en las consideraciones de Albers (2003), consideramos a las administraciones de justicia como unidades productoras de "asuntos resueltos", que será nuestro output ${ }^{12}$, para lo cual necesita de capital, representado por el "gasto en justicia", y trabajo, que aproximaremos por el "número de jueces y fiscales” considerados todos ellos como inputs, a los que añadiremos el "número de asuntos ingresados y pendientes" como proxy de los bienes y servicios utilizados para la obtención del output ${ }^{13}$.

Respecto al trabajo seminal de Pedraja y Salinas (1995), coincidimos en la elección del output, aunque el ámbito geográfico de este trabajo nos impide descomponerlo en la forma en que estos autores lo hacen: de dicha descomposición en asuntos resueltos mediante sentencia y los finalizados sin resolución de fondo no disponemos de información. Respecto al input "número de jueces y fiscales", coincidimos con el trabajo de Kittelsen y Førsund (1992) en cuanto a la consideración de lo que constituye los componentes del staff establecido por el Estado, evitando la consideración de otras figuras, como los funcionarios judiciales, que podrían distorsionar los datos ya que, en el caso de España, por ejemplo, no dependen de la administración de justicia estrictamente sino de las comunidades autónomas que tienen.

En el cuadro 2 recogemos los países considerados en el estudio ${ }^{14}$ y las definiciones de los outputs e inputs utilizados.

Y ofrecemos en el cuadro 3 algunos estadísticos descriptivos relativos al output y a los inputs.

En ella observamos la gran dispersión que muestran los inputs y outputs, fundamentalmente en el número de asuntos resueltos. Respecto al personal, constatamos en una primera perspectiva, las grandes diferencias que existen entre los medios puestos a disposición de las administraciones de justicia de los distintos países del entorno europeo, oscilando el número de jueces y fiscales por cada 100.000 habitantes entre los casi 60 de Croacia y no llegando a 9 en Inglaterra-Gales.

\footnotetext{
12 En esta investigación solo se están computando los asuntos de la primera instancia y de naturaleza penal, civil-comercial, contenciosa y administrativa, porque no todos los países ofrecen los datos de la segunda o ulteriores instancias, de los asuntos no contenciosos de naturaleza civil-comercial, de los relativos a la ejecución y de los registros de la propiedad y comercio.

${ }^{13}$ Vincovà (2005) resalta la importancia de la elección adecuada de inputs y outputs para una determinación correcta de la eficiencia.

${ }^{14}$ Los países considerados son aquellos de los que se disponen datos de los inputs y outputs elegidos.
} 


\begin{tabular}{|c|c|c|c|c|c|}
\hline \multicolumn{6}{|c|}{ CUADRO 2. PAÍSES, OUTPUT E INPUTS CONSIDERADOS EN EL ESTUDIO } \\
\hline \multirow{7}{*}{ Países } & Albania & Andorra & Austria & Azerbaiyán & $\begin{array}{l}\text { Bosnia- } \\
\text { Herzegovina }\end{array}$ \\
\hline & Croacia & Rep. Checa & Dinamarca & Estonia & Finlandia \\
\hline & Georgia & Alemania & Hungría & Italia & Letonia \\
\hline & Lituania & Malta & Moldavia & Montenegro & Noruega \\
\hline & Polonia & Portugal & Rumania & Fed. Rusia & Serbia \\
\hline & Eslovaquia & Eslovenia & España & Suecia & Suiza \\
\hline & Macedonia & Turquía & Ukraine & $\begin{array}{l}\text { Inglaterra- } \\
\text { Gales }\end{array}$ & \\
\hline Output & \multicolumn{2}{|c|}{ Número de Asuntos resueltos } & \multicolumn{3}{|c|}{$\begin{array}{l}\text { Los asuntos resueltos o terminados son aquellos } \\
\text { que han finalizado en el año que se contabiliza. } \\
\text { El procedimiento se entiende finalizado bien } \\
\text { porque ha concluido definitivamente (sentencia } \\
\text { firme o archivo definitivo) o provisionalmente } \\
\text { (archivo provisional) o porque ha pasado a la } \\
\text { segunda instancia en virtud de recurso. }\end{array}$} \\
\hline \multirow{3}{*}{ Inputs } & \multicolumn{2}{|c|}{$\begin{array}{l}\text { Número de Asuntos ingresados + } \\
\text { número de asuntos pendientes }\end{array}$} & \multicolumn{3}{|c|}{$\begin{array}{l}\text { Solo se han tenido en cuenta los asuntos ingresados } \\
\text { nuevos. } \\
\text { Los asuntos pendientes o en trámite (también de la } \\
\text { primera instancia de naturaleza penal, civil y } \\
\text { comercial contenciosa, y administrativa) son } \\
\text { aquellos asuntos ingresados de ese año o años } \\
\text { anteriores que aún no han sido resueltos y, por } \\
\text { tanto, aún están en tramitación a fecha } 1 \text { de enero } \\
\text { de } 2012 \text {. }\end{array}$} \\
\hline & \multicolumn{2}{|c|}{ Gasto público $(€)$} & \multicolumn{3}{|c|}{$\begin{array}{l}\text { Presupuesto del Estado para la Administración de } \\
\text { Justicia. }\end{array}$} \\
\hline & \multicolumn{2}{|c|}{$\begin{array}{l}\text { Personal: Número de jueces + } \\
\text { número de fiscales }\end{array}$} & \multicolumn{3}{|c|}{$\begin{array}{l}\text { Juez: La autoridad encargada de tomar o de } \\
\text { participar una decisión judicial a partes } \\
\text { enfrentadas que pueden ser personas físicas o } \\
\text { jurídicas, en el curso de un proceso. } \\
\text { Fiscal/Ministerio Público }{ }^{15} \text { : La autoridad } \\
\text { encargada de velar, en nombre de la sociedad y el } \\
\text { interés general, por la aplicación de la ley cuando } \\
\text { ello está penalmente sancionado, teniendo en } \\
\text { cuenta, de una parte, los derechos de los individuos } \\
\text { y, de otra, la necesaria eficacia del sistema de } \\
\text { justicia penal. }\end{array}$} \\
\hline
\end{tabular}

\footnotetext{
${ }^{15}$ Recomendación REC 2000 (19) del Comité de Ministros del Consejo de Europa de 6 de octubre de 2000.
} 


\begin{tabular}{|c|c|c|c|c|c|}
\hline \multicolumn{1}{|c|}{ CUADRO 3. ESTADÍSTICOS DESCRIPTIVOS DEL OUTPUT Y DE LOS INPUTS } \\
\hline & Media & $\begin{array}{c}\text { Coefic. } \\
\text { de } \\
\text { variación } \\
(\%)\end{array}$ & Mediana & Máximo & Mínimo \\
\hline $\begin{array}{c}\text { Número de asuntos } \\
\text { resueltos (Output) }\end{array}$ & $1.205 .557,97$ & 207,87 & $236.296,5$ & 13.687 .375 & 5.310 \\
\hline $\begin{array}{c}\text { Número de asuntos } \\
\text { ingresados y pendientes a } \\
\text { 1 de enero de 2012 (Input) }\end{array}$ & $1.722 .878,38$ & 178,57 & 371.532 & 14.676 .826 & 9.205 \\
\hline $\begin{array}{c}\text { Gasto en Justicia } \\
\text { (€)(Input) }\end{array}$ & $11.599 .21 .477,35$ & 174,45 & $402.025 .569,5$ & 9.170 .186 .780 & 6.442 .382 \\
\hline $\begin{array}{c}\text { Jueces y fiscales(Input) } \\
\text { Jueces y fiscales/100.000 } \\
\text { hab. }\end{array}$ & $6.131,23529$ & 197,06 & $2.002,5$ & 65.877 & 28 \\
\hline \begin{tabular}{c} 
Fuente: Elaboración propia. \\
\hline
\end{tabular} & 32,87 & 234,91 & 32,7 & 59,81 & 8,32 \\
\hline
\end{tabular}

\section{RESULTADOS}

En el cuadro 4 se muestran las eficiencias relativas obtenidas ${ }^{16}$ para la administración de justicia de cada uno de los países considerados, con el modelo CCR orientado output ${ }^{17}$.

De los 34 países analizados, 27 han resultado ser ineficientes. España se encuentra entre los 7 países que han resultado ser eficientes. Mostramos los estadísticos correspondientes a las puntuaciones en el cuadro 5, de la que inferimos que existe escasa variabilidad entre las puntuaciones obtenidas por las distintas administraciones de justicia, ocurriendo que el 50 por 100 de ellas presenta un nivel de ineficiencia inferior a 0,17 y un 25 por 100 de ellas niveles de ineficiencia menores al $0,044 .^{18}$.

Hay que indicar que el modelo utilizado asigna puntuación máxima, un 1, no solo a las que resultan eficientes en su comparación con otras, sino también a todas aquellas unidades productivas que no han podido ser comparada con ninguna otra (Ganley y Cubbin, 1992). Esto es lo que ha ocurrido con las siete administraciones de justicia que han resultado eficientes, no han podido ser comparadas con ninguna de las otras unidades consideradas y el modelo las sitúa en la frontera eficiente. Ahora bien, como señala Ley (1991) cuando a una unidad se le asigna una puntuación 1 porque únicamente se ha comparado consigo misma, su eficiencia resulta "sospechosa". En tal caso, la frecuencia con que estas unidades eficientes aparecen como referentes de las ineficientes es un buen criterio, aunque no el único (Akbarian, 2015), para delimitar su verdadera condición de eficiencia. Si este número es elevado, la unidad correspondiente es genuinamente eficiente con respecto a un buen número de unidades. En nuestro caso, de las 27 posibilidades, 34 unidades menos las 7 que solo son comparadas consigo misma, la correspondiente a la Federación Rusa se ha utilizado como referencia para 24 países, apareciendo claramente destacada. España ha servido como

\footnotetext{
${ }^{16}$ Software DEAP.

${ }^{17}$ Consideramos el modelo orientado al output ya que tratamos de conseguir el máximo output con los inputs disponibles.

${ }^{18}$ Estas puntuaciones pueden utilizarse de distintas formas para obtener una ordenación de los países (Adler et al., 2002).
} 
referencia solo para Italia, luego su puntuación de eficiencia máxima queda significativamente matizada.

\section{CUADRO 4. EFICIENCIAS RELATIVAS (MODELO CCR)}

\begin{tabular}{|l|l|}
\hline \multicolumn{1}{|c|}{ País } & $\begin{array}{l}\text { Eficiencia técnica } \\
\text { relativa }\end{array}$ \\
\hline Albania & 0,724 \\
\hline Andorra & 0,722 \\
\hline Austria & 0,794 \\
\hline Azerbaiyán & 0,93 \\
\hline Bosnia- & 0,954 \\
\hline Croacia & 0,824 \\
\hline Rep. Checa & 0,859 \\
\hline Dinamarca & 0,965 \\
\hline Estonia & 0,823 \\
\hline Finlandia & 0,724 \\
\hline Georqia & 0,855 \\
\hline Alemania & 0,704 \\
\hline Hunqría & 0,83 \\
\hline Italia & 0,927 \\
\hline Letonia & 0,665 \\
\hline Lituania & 0,865 \\
\hline Malta & 1 \\
\hline Moldavia & 1 \\
\hline Monteneqro & 0,653 \\
\hline Norueqa & 0,843 \\
\hline Polonia & 0,777 \\
\hline Portuqal & 0,586 \\
\hline Rumania & 0,91 \\
\hline Fed. Rusia & 1 \\
\hline Serbia & 0,606 \\
\hline Eslovaquia & 0,525 \\
\hline Eslovenia & 0,654 \\
\hline España & 1 \\
\hline Suecia & 0,778 \\
\hline Suiza & 0,781 \\
\hline Macedonia & 1 \\
\hline Turquía & 1 \\
\hline Ukrania & 0,923 \\
\hline Inqlaterra-Gales & \\
\hline
\end{tabular}

Fuente: Elaboración propia. 
CUADRO 5. ESTADÍSTICOS DESCRIPTIVOS DE LAS PUNTUACIONES

DE EFICIENCIA (MODELO CCR)

\begin{tabular}{|l|l|}
\hline \multicolumn{2}{|c|}{ Puntuaciones eficiencia técnica } \\
\hline Media & 0,8294 \\
\hline Coeficiente de variación & $16,589 \%$ \\
\hline Mediana & 0,8365 \\
\hline Percentil 25 & 0,9567 \\
\hline Percentil 75 & 0,7235 \\
\hline
\end{tabular}

Fuente: Elaboración propia.

Si repetimos el análisis relajando la suposición de rendimiento constante, modelo BCC, podemos detectar aquellos países cuya administración de justicia no está trabajando a una escala óptima, pudiendo ofrecer una medida de la eficiencia técnica pura. En el cuadro 6 presentamos los resultados.

El cociente entre los índices de eficiencia CCR y BCC, refleja la eficiencia de escala ${ }^{19}$. De los resultados obtenidos observamos que, a las unidades eficientes detectadas en el modelo CCR se ha unido Andorra que ha pasado a ser eficiente y es la más utilizada como referencia para las otras, en concreto de las 26 ineficientes, 25 de ellas utilizan, entre otros, a este país como referente. Además, con la nueva información proporcionada por el modelo BCC, observamos que, de las unidades ineficientes del modelo CCR, tres de ellas, Italia, Croacia y Rumania, presentan rendimientos a escala decrecientes, y las 24 restantes rendimientos a escala crecientes, existiendo una elevada correlación entre los índices de eficiencia de ambos modelos $(93,4336 \%)$.

${ }^{19}$ Banker y Thrall (1992). 


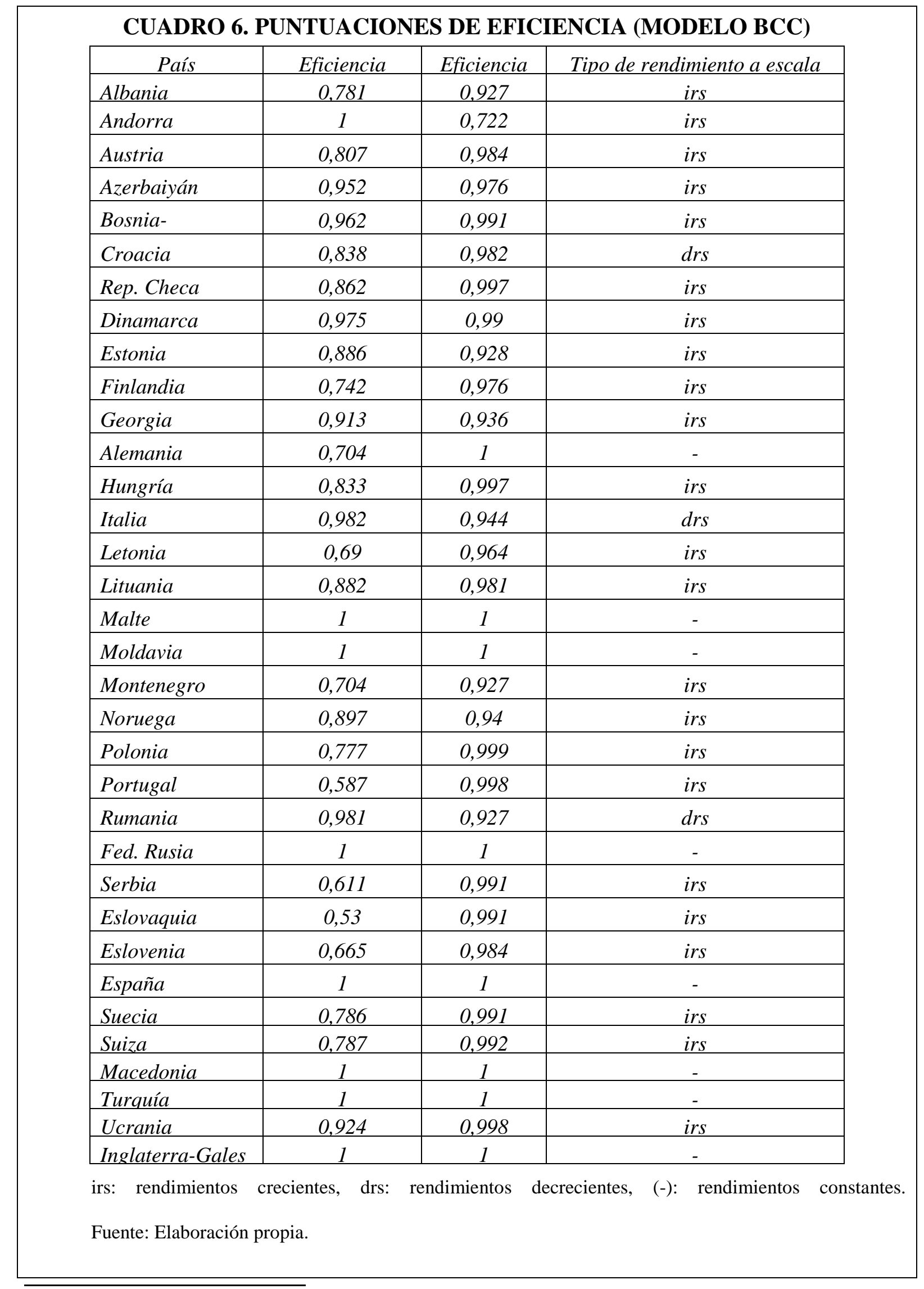

${ }^{20}$ Eficiencia de escala= Eficiencia CCR/Eficiencia BCC 


\section{RELACIONES ENTRE LAS INEFICIENCIAS Y EL GASTO PÚBLICO}

En este apartado veremos si, como se aduce desde algunos colectivos, el gasto en justicia condiciona el mejor o peor funcionamiento de la misma. Para ello, y basándonos en los resultados obtenidos en el apartado anterior, vamos a relacionar la puntuación de eficiencia de la administración de justicia con el gasto presupuestado para la misma. Vamos a medir este gasto mediante tres indicadores:

- porcentaje de gasto en la administración de justicia respecto al gasto total del Estado

- gasto en la administración de justicia por habitante, medido en euros

- número de jueces y fiscales por cada 100.000 habitantes

que miden, de diferente forma, lo que podríamos considerar como el esfuerzo en la administración de justicia que realiza un país.

A continuación presentamos algunas características de los tres indicadores elegidos. En primer lugar, en el cuadro 7 mostramos algunos de sus estadísticos.

\begin{tabular}{|c|c|c|c|}
\hline \multicolumn{4}{|c|}{$\begin{array}{l}\text { CUADRO 7. ESTADÍSTICOS DESCRIPTIVOS DE LOS INDICADORES DE } \\
\text { GASTO CONSIDERADOS }\end{array}$} \\
\hline & $\begin{array}{c}\text { Porcentaje de gasto en } \\
\text { Justicia respecto al } \\
\text { gasto total del Estado }\end{array}$ & $\begin{array}{l}\text { Gasto en Justicia } \\
\text { por habitante }\end{array}$ & $\begin{array}{l}\text { Número de jueces y } \\
\text { fiscales por cada } \\
100.000 \text { habitantes }\end{array}$ \\
\hline Media & $1,05 \%$ & $54,11 €$ & 32,867 \\
\hline $\begin{array}{l}\text { Coeficiente de } \\
\text { variación }\end{array}$ & $197,79 \%$ & $132,07 \%$ & $234,91 \%$ \\
\hline Mediana & $0,9 \%$ & $43,56 €$ & 32,715 \\
\hline Percentil 25 & $0,707 \%$ & $27,13 €$ & 22,37 \\
\hline Percentil 75 & $1,31 \%$ & $78,997 €$ & 43,25 \\
\hline Máximo & $2,58 \%$ & $198,82 €$ & \\
\hline Mínimo & $0,37 \%$ & $4,68 €$ & \\
\hline
\end{tabular}

Lo más llamativo del cuadro es la fila correspondiente al coeficiente de variación, que indica la alta disparidad existente entre los países en los indicadores considerados.

Encontramos, además, mediante el test de Kolmogorov-Smirnov, que ninguna de las tres variables sigue una distribución normal, y mediante la prueba de Friedman para muestras relacionadas, rechazamos la igualdad de distribuciones para las tres variables a un nivel de significación del 5 por 100.

En el gráfico 1 se sitúan los países en el plano "porcentaje de gasto en administración de justicia con respecto al gasto del Estado - gasto en administración de justicia por habitante”. 


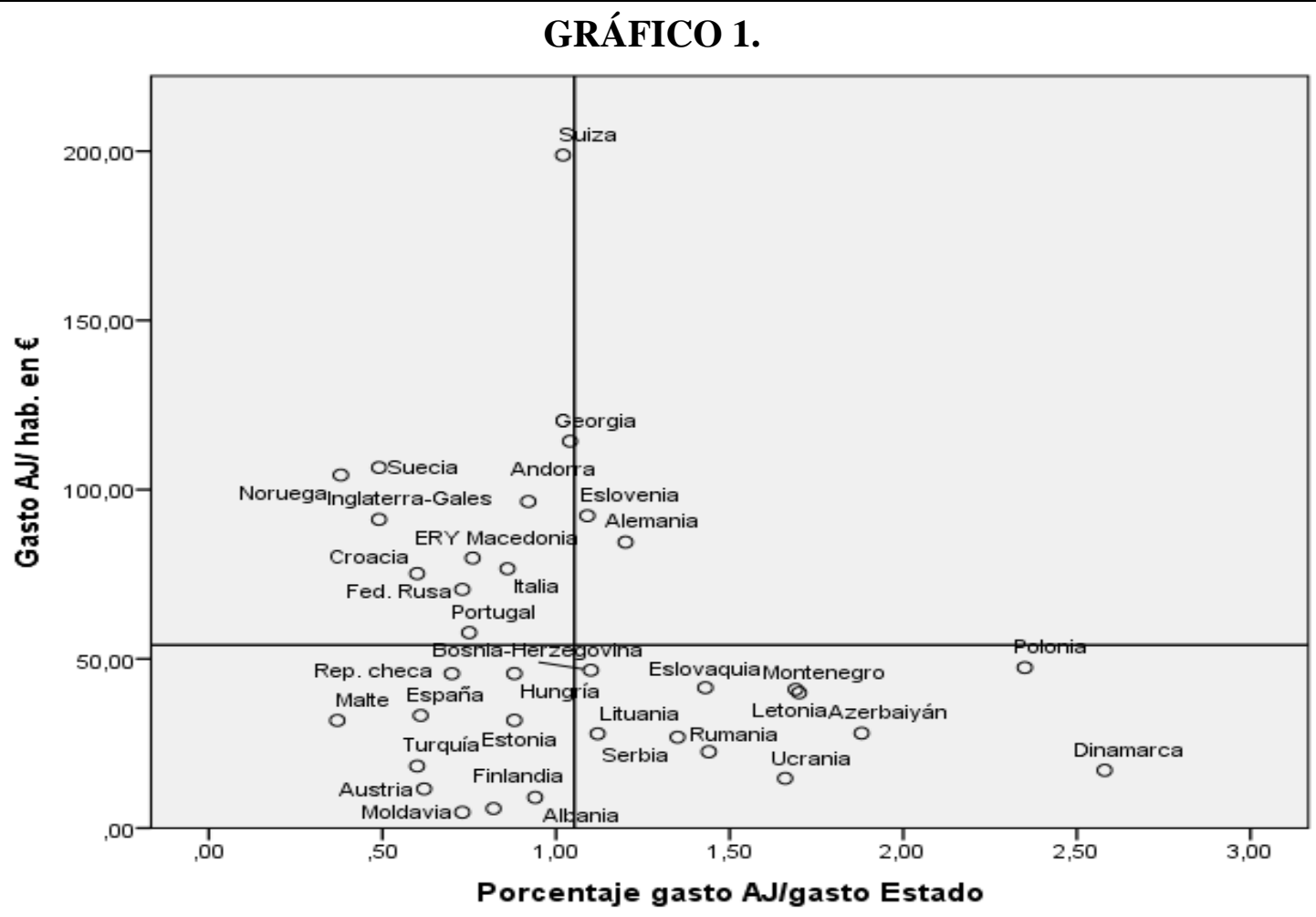

Fuente: Elaboración propia. (Software SPSS).

Encontrando que los 11 en el cuadrante inferior derecho, con alto porcentaje de gasto en justicia con respecto al gasto del Estado, no se traduce en mayor gasto en justicia por habitante. En el extremo opuesto se sitúan otros 10. España se encuentra en el cuadrante inferior izquierdo: De los menores porcentajes de gasto en justicia con respecto al gasto del Estado y menores en gasto en justicia por habitante.

De hecho los coeficientes de correlación, cuadro 8, nos muestran cómo el porcentaje de gasto en la administración de justicia respecto al gasto total del Estado guarda una relación negativa con el gasto en la administración de justicia por habitante, $y$, sin embargo, es positiva con el número de jueces y fiscales.

\begin{tabular}{|l|l|l|l|}
\hline \multicolumn{5}{|c|}{ CUADRO 8. CORRELACIONES } \\
\hline \multicolumn{1}{|c|}{ Variables } & $\begin{array}{l}\text { Gasto en AJ/gasto } \\
\text { Estado }(\%)\end{array}$ & $\begin{array}{l}\text { Gasto AJ por } \\
\text { habitante }(€)\end{array}$ & $\begin{array}{l}\text { Jueces y fiscales por } \\
\text { cada } \\
\text { habitantes }\end{array}$ \\
\hline gasto en AJ/gasto Estado $(\%)$ & $1,000\left(0,000^{a}\right)$ & $-0,240(0,162)$ & $0,534(0,124)$ \\
\hline Gasto AJ por habitante $(€)$ & $-0,240(0,162)$ & $1,000(0,000)$ & $-0,213(0,164)$ \\
\hline $\begin{array}{l}\text { Jueces y fiscales por cada } \\
100.000 \text { habitantes }\end{array}$ & $0,534(0,124)$ & $-0,213(0,164)$ & $1,000(0,000)$ \\
\hline $\begin{array}{l}\text { F }\left(^{a}\right) \text { : Entre paréntesis la desviación estándar. } \\
\text { Fuente: Elaboración propia (Calculadas con el paquete R Héctor). }\end{array}$ \\
\hline
\end{tabular}


Para el análisis de las relaciones con las puntuaciones de eficiencia, y dado que estas son relativas, es decir, están en relación entre ellas, y con valores comprendidos entre 0 y 1 , hemos hecho lo mismo con los tres indicadores, de tal forma que se han relacionado utilizando la expresión:

$$
I_{i}^{n}=\frac{I_{i}-\operatorname{Min}_{i}\left(I_{i}\right)}{\operatorname{Max}_{i}\left(I_{i}\right)-\operatorname{Min}_{i}\left(I_{i}\right)}
$$

Denotando el subíndice $i$ al país de referencia, $I_{i}$ el indicador en justicia utilizado e $\mathrm{I}^{\mathrm{n}}$, $\mathrm{n}=1,2,3$ el indicador relativizado. De esta forma al país que toma el mayor valor para el índice se le asigna un $1 \mathrm{y}$ al que toma el menor valor un 0 . El resto de los países quedan situados dentro de esta escala, correspondiendo un mayor valor con un mayor esfuerzo en el gasto.

En los gráficos 2 y 3 se visualizan las relaciones entre las puntuaciones de eficiencia obtenidas, modelos CCR y BCC respectivamente, y los indicadores considerados.

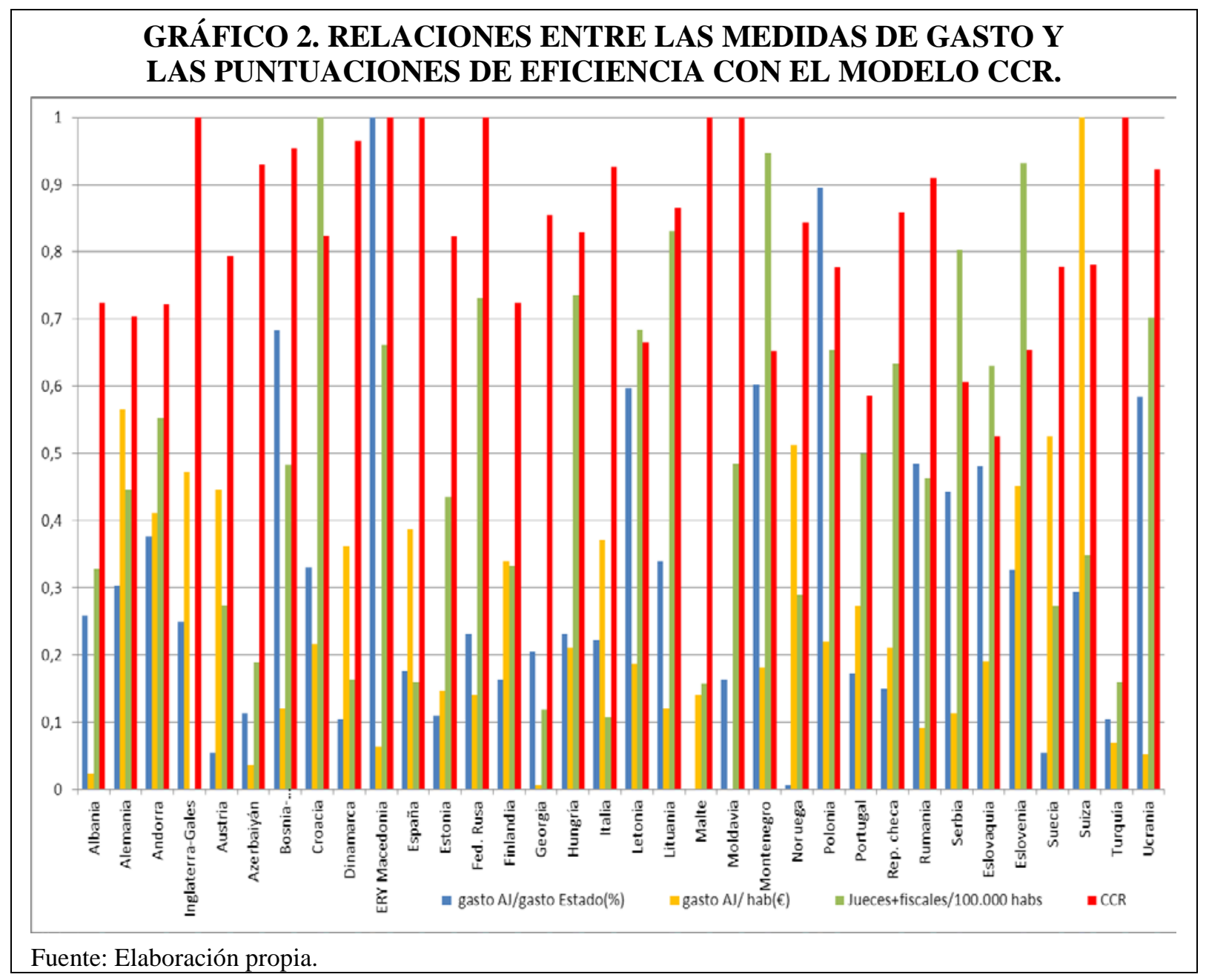




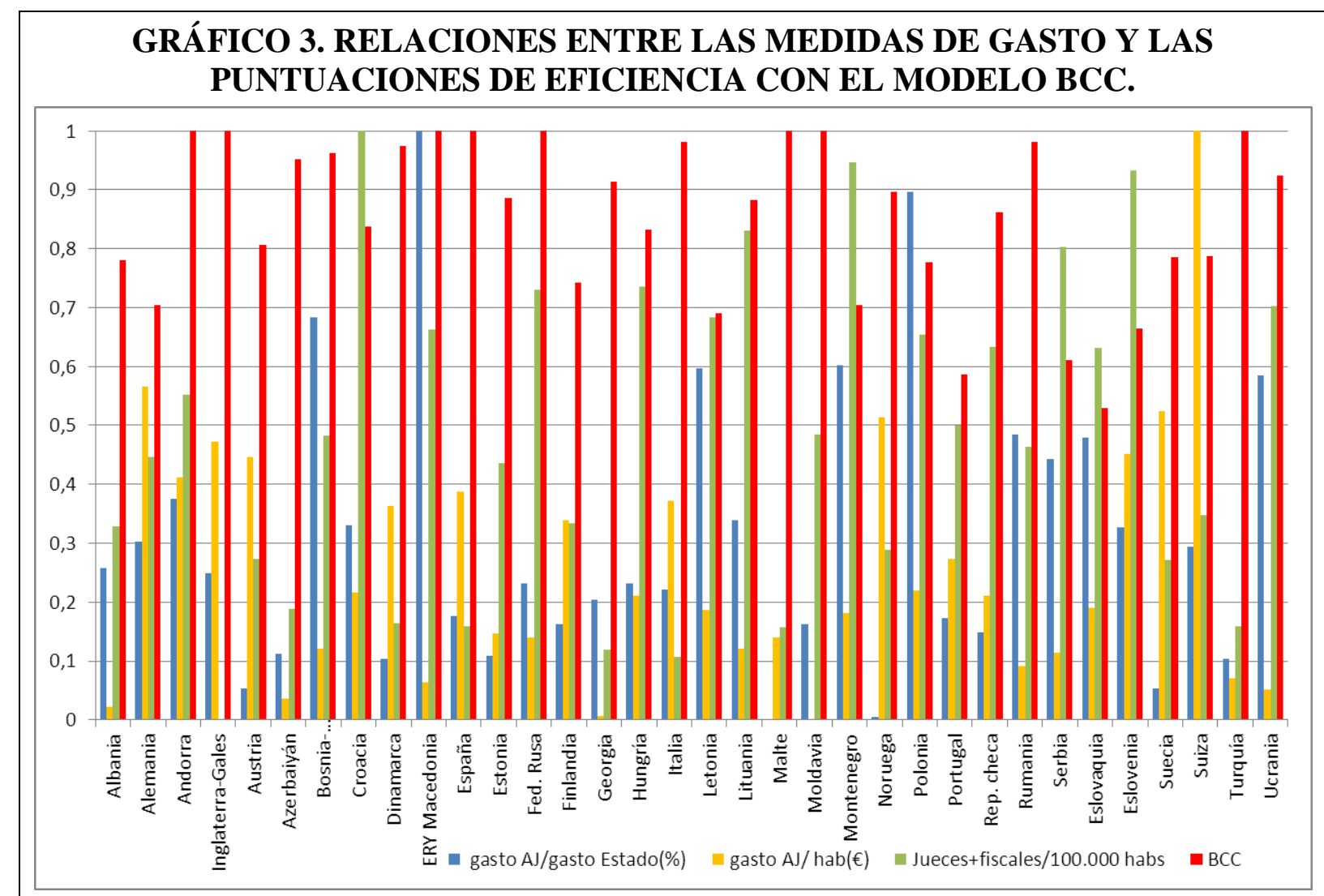

Fuente: Elaboración propia.

A través de modelos tobit (Tobin, 1958) formalizamos las relaciones entre las variables.

\begin{tabular}{|c|c|c|c|}
\hline \multicolumn{4}{|c|}{ CUADRO 9. REGRESIONES TOBIT ${ }^{21}$} \\
\hline $\begin{array}{l}\text { Modelo } \\
\text { estimado }\end{array}$ & $\begin{array}{l}\text { Ecuación y Estadístico z de } \\
\text { significatividad del coeficiente }\end{array}$ & $\begin{array}{c}\text { Bondad del ajuste } \\
\text { (Estadístico Chi- } \\
\text { cuadrado) }\end{array}$ & $\begin{array}{l}\text { Intervalos de confianza de } \\
\text { los coeficientes al 95\% }\end{array}$ \\
\hline \multirow{3}{*}{ CCR } & $\begin{array}{l}C C R=1,91 * \text { Gto. AJ/Estado } \\
(0,30)^{a} z=6,36(p<0,00001)\end{array}$ & $\begin{array}{l}30,09 \\
(p=2,9 e-007)\end{array}$ & $(1,322,2,501)$ \\
\hline & $\begin{array}{l}C C R=2,06 * \text { Gto. AJ/hab. } \\
(0,36)^{a} z=5,7(p<0,00001)\end{array}$ & $\begin{array}{l}24,29 \\
(p=5,3 e-006)\end{array}$ & $(1,352,2,760)$ \\
\hline & $\begin{array}{l}C C R=1,36 * \text { Jueces y fiscales }(0,18)^{a} \\
z=7,5(p<0,00001)\end{array}$ & $\begin{array}{l}32,18 \\
(p=1,02 e-007)\end{array}$ & $(1,002,1,716)$ \\
\hline \multirow{3}{*}{$B C C$} & $\begin{array}{l}\text { BCC }=2,02 * \text { Gto. AJ/Estado } \\
(0,32)^{a} z=6,3(p<0,00001)\end{array}$ & $\begin{array}{l}28,57 \\
(p=6,25 e-007)\end{array}$ & $(1,395,2,646)$ \\
\hline & $\begin{array}{l}B C C=2,19 * \text { Gto. AJ } / \text { hab. } \\
\left.(0,38)^{a} z=5,8 p<0,00001\right)\end{array}$ & $\begin{array}{l}20,68 \\
(p=3,24 e-005)\end{array}$ & $(1,446,2,940)$ \\
\hline & $\begin{array}{l}\text { BCC }=1,43^{*} \text { Jueces y fiscales } \\
(0,19)^{a} z=7,4(p<0,00001)\end{array}$ & $\begin{array}{l}32,06 \\
(p=1,09 e-007)\end{array}$ & $(1,049,1,807)$ \\
\hline
\end{tabular}

${ }^{21}$ Software SPSS. 
En primer lugar constatamos, mediante los estadísticos Chi cuadrado, que todos los modelos han resultado significativos a niveles superiores al 99 por 100. De la misma forma, observamos que los resultados para cada modelo, CCR y BCC, difieren levemente, pues ya vimos que ambas puntuaciones estaban correlacionadas a un nivel superior al 93 por 100 . Además, en cada uno de los modelos con un solo coeficiente, este ha resultado ser significativo, usando el estadístico z, a niveles también superiores al 99 por 100. Y para ambos casos (CCR y BCC) las puntuaciones dependen en mayor medida del esfuerzo de gasto medido a través del porcentaje por habitante del gasto en la administración de justicia. Es decir, si el país se posiciona mejor en este indicador con respecto a la posición de otros países, resultará aumentada su eficiencia relativa.

Este resultado coincide con la conclusión que extraen Palumbo et al. (2013). Y aunque a simple vista se contradice con los obtenidos por otros autores como Cross y Donelson (2010) y Voigt y El Bialy (2012), que concluyen que la eficiencia judicial no es función del presupuesto, hay que tener en cuenta dos hechos fundamentales: en primer lugar, el concepto de eficiencia que utilizan no coincide con el nuestro, no medimos una eficiencia absoluta sino que asignamos una puntuación que relaciona la de cada país con la del resto del conjunto de países considerados; en segundo lugar, usan la definición de la variable presupuesto tal como la define la CEPEJ (cociente entre el presupuesto público aprobado y el PIB) mientras que en este trabajo la variable presupuesto se utiliza, en valores absolutos para determinar la eficiencia relativa de cada país y, a efectos de relacionarla con dicha eficiencia, se transforma para que refleje, no ya el presupuesto dedicado, sino el esfuerzo de gasto que realiza el país en relación con el esfuerzo que hacen los otros países considerados.

\section{CONCLUSIONES}

La evaluación de las administraciones de justicia no es tarea fácil debido a la variedad de sistemas y prácticas judiciales de los países europeos. La CEPEJ trabaja desde su creación en la definición de indicadores "judiciales" válidos para todos y que puedan permitir comparaciones entre ellos. El problema se agrava cuando se trata de poner en relación estos indicadores con el gasto que cada país realiza en su justicia, aún más si esta está descentralizada, como ocurre en algunos países entre los que se encuentra España. Esta circunstancia no debe servir como excusa para no someter a las administraciones de justicia a una evaluación continua, exigiéndoles, como servicio público que son y por las enormes repercusiones en la vida social y económica de los ciudadanos, una adecuada utilización de sus recursos, a la vez que proponiendo, en la medida de lo posible, acciones correctoras.

Es, en esta línea en la que se encuadra el presente trabajo. Conscientes de sus muchas limitaciones, esta investigación, que constituye una primera aproximación al análisis de la eficiencia con la que actúa la administración de justicia en España, en su comparativa europea, ha mostrado que, en este contexto, su posición es atípica, en el sentido de que la utilización de los recursos considerados, con los que se atiende a una de las principales demandas (el número de asuntos resueltos), no es comparable a los que se obtiene en otros países con actuaciones similares. Si bien es cierto que solo se han tratado los correspondientes a la primera instancia de naturaleza penal, civil y comercial contenciosa, y administrativa, y sería deseable un estudio más detallado considerando separadamente los distintos tipos de causas.

Del segundo tipo de análisis realizado, la relación entre la eficiencia técnica de la administración de justicia y el gasto realizado en la misma, los análisis muestran que existe una relación positiva y significativa entre ellos, tanto si se mide el gasto a través del 
Porcentaje de gasto en la administración de justicia como porcentaje del gasto total del Estado, como si se mide por el gasto realizado por habitante, como si utilizamos el número de jueces y fiscales por cada 100.000 habitantes. Concluimos que el esfuerzo de gasto que realiza un país en su administración de justicia influye positivamente en los resultados obtenidos, en mayor medida si se mide este esfuerzo a través del gasto por habitante que se realiza en la justicia.

\section{BIBLIOGRAFÍA}

Akbarian, D. (2015): "Ranking All DEA-Efficient DMUs Based on Cross Efficiency" Journal of Optimization, Article ID 594727, 10 pages.

Adler, N., Friedman, L. y Sinuany-Stern, Z. (2002): "Review of ranking methods in the data envelopment analysis context”, European Journal of Operational Research, 140(2), 249-265.

Albers, P. (2003): "Evaluating Judicial Systems: 'A Balance between variety and generalization”, European Commission for the Efficiency of Justice ( CEPEJ ), Councils for the Judiciary in EU Countries.

Ballbé, M. y Padrós, C. (1997): Estado competitivo y armonización europea, Ariel, Barcelona.

Banker R.D., Charnes, A. y Cooper, W. (1984): "Some Models for Estimating Technical and Scale Inefficiencies in Data Envelopment Analysis”, Management Science, 30(9), 1078-1092.

Banker, R. D. y Thrall, R. M. (1992): "Estimation of Returns to Scale Using Data Envelopment Analysis”, European Journal of Operational Research, 62(1), 74-84.

Barómetros de opinión. Centro de Estudios de Investigaciones Sociológicas. http://www.cis.es/cis/export/sites/default/-

Archivos/Indicadores/documentos_html/TresProblemas.html.

CEPEJ (European Commission for the Efficiency of Justice) (2012): European judicial systems: Efficiency and quality of justice, 2010 data. Council of Europe Publishing, Strasbourg.

Charnes, A., Cooper, W.W. y Rhodes, E. (1978): "Measuring the efficiency of decision making units”, European Journal Operational Research, 2(6), 429-444.

Círculo de Empresarios. (2003): Justicia, Economía y Empresa, Documentos Círculo.

Coelli, T. et al. (2005): An Introduction to efficiency and productivity analysis, Springer.

Council Of Europe. Committee Of Ministers. Resolution Res (2002)12. Disponible en http://www.coe./dghl/cooperation/cepej/presentation/cepej_en.asp

Cross, F. B. y Donelson, D. C. (2010): “Creating Quality Courts”, Journal of Empirical Legal Studies, 7 (3), 490-510.

Deynely, F. (2012): “Analysis of relationship between efficiency of justice services and salaries of judges with two-stage DEA method", European Journal of Law and Economics, 34, 477-493.

El-Mahgary, S., Rönnholm, P., Hyyppä, H., Haggrén, H. y Koponen, J. (2014): “Evaluating the performance of university course units using data envelopment analysis", Cogent Economics \& Finance, 2: 918856. 
Fabbri, D. (2010): “Law Enforcement and Firm Financing: Theory and Evidence”, Journal of the European Economic Association, 8, (4), 776-816.

Farrell, M. J. (1957):” The measurement of productive efficiency”, Journal of the Royal Statistical Society Series A, 120, 253-281.

Finocchiaro, M. y Guccio, C. (2014): "Searching for the source of technical inefficiency in Italian judicial districts: an empirical investigation”, European Journal of Law and Economics, 38 (3) 369-391.

Ganley, J.A. y Cubbin, J.S. (1992): Public Sector Efficiency Measurement: Applications of Data Envelopment Analysis, Elsevier, North-Holland.

García Posada, M. y Mora Sanguinetti, J.S. (2013): "Firm Size and Judicial Efficacy: Evidence for the New Civil Procedures in Spain”, Documento de Trabajo 1303, Banco de España, Eurosistema.

García Rubio, M.A. y Rosales López, V. (2010): “Justicia y Economía: Evaluando la Eficiencia Judicial en Andalucía”, Indret.

Hayo, B. y S. Voigt, S. (2014): “The Relevance of Judicial Procedure for Economic Growth”, CESifo Economic Studies, 60, (3), 490-524.

Iglesias, P. y Arias, X.C. (2006): "Transaction Costs and Efficiency of the Spanish Judicial System”, ISLE Conference, Roma.

Ippoliti, R., Melcarne, A. y Ramello, G. B. (2015): “The Impact of Judicial Efficiency on Entrepreneurial Action: A European Perspective”, Economic Notes, 44, 57-74.

Jimeno, J.F., Martínez-Matute, M. y Mora Sanguinetti, J.S. (2015): “Employment protection legislation and labor court activity in Spain”, Banco de España. Documentos de Trabajo, N. ${ }^{0} 1507$

Kittelsen, S. y Førsund, F. R, (1992): "Efficiency analysis of Norwegian district courts”,Journal of Productivity Analysis, 3, 3, 277-306.

Ley, E. (1991): "Eficiencia productiva: Un estudio aplicado al sector hospitalario", Investigaciones Económicas, 15 (1), 71-88.

Liu, J.S., Lu, L., Wen-MinLu y Lin, B.

(a)(2013): "Data envelopment analysis 1978-2010: A citation-based literature survey", Omega, 41, 3-15.

(b)(2013): “A survey of DEA applications”, Omega, 41, 893-902.

Mar Molinero, C. y Woracker, D. (1996): “Data Envelopment Analysis: - a non-mathematical introduction", OR Insight 9 (4).

Ménard C. y Du Marais, B. (2008): “Can we rank legal systems according to their economic efficiency?”, Washington University Journal of Law \& Policy: Law \& The New Institutional Economics, 26, 55- 80.

Mora-Sanguinetti, J. S. (2016): "Evidencia reciente sobre los efectos económicos del funcionamiento de la justicia en España”, Boletín Económico-Banco de España, 1, 3341.

Mora-Sanguinetti, J.S., Martínez-Matute, M. y M. García-Posada (2016). “Credit, crisis and contract enforcement: evidence for the Spanish loan market”, Working Papers, 1630. Banco de España. 
Nazarko, J. y Šaparauskas, J. (2014): “Application of DEA method in efficiency evaluation of public higher education institutions”, Technological and Economic Development of Economy, 20(1), 25-44.

Nieto, A. (2005): El desgobierno judicial, Fundación Alfonso Martín Escudero, Editorial Trotta. Madrid.

Padilla, J., Llorens, V., Pereiras, S. y N. Watson. (2007): "Eficiencia judicial y eficiencia económica: el mercado crediticio español”, en La Administración Pública que España necesita, Libro Marrón, Círculo de Empresarios, Madrid.

Palečková, I. (2015): "Estimation of banking efficiency determinants in the Czech Republic", Journal of Applied Economic Sciences, 10(2), 234-242.

Palumbo, G., Guipponi, G., Nunziana, L. y Mora Sanguinetti, J.S. (2013): “The Economics of Civil Justice: New Cross-country Data and Empirics”, OECD Economics Department Working Papers, No. 1060, OECD Publishing. http://dx.doi.org/10.1787/5k41w04ds6kf-en.

Pastor Prieto, S. (1989): Sistema Jurídico y Economía. Una introducción al Análisis Económico del Derecho, Ed. Tecnos.

Pastor Prieto, S.

(a) (2003): "Dilación, eficiencia y costes”, Foro sobre la Reforma y Gestión de la Justicia, Fundación BBVA, Bilbao.

(b) (2016): Análisis Económico de la Justicia y Reforma Judicial, Editorial Tirant lo Blanch.

Pastor Prieto, S. y Rosales, V. (2005): "Dos dimensiones de la eficacia de la justicia”, Economistas, 105.

Pedraja, F. y Salinas, J. (1995): "La eficiencia en la Administración de Justicia: Las Salas de lo contencioso de los Tribunales Superiores de Justicia”, Revista de Economía Aplicada, 8, (III), 163-195.

Peyrache A. y Zago, A. (2016): "Large courts, small justice! The inefficiency and the optimal structure of the Italian justice sector”, Omega, 64, 42-56.

Rosales López, V. (2008): "Economics of court performance: an empirical analysis", European Journal of Law and Economics, 25, 231-251.

Santos, S.P. y Amado, C.A.F. (2014): "On the need for reform of the Portuguese judicial system - Does Data Envelopment Analysis assessment support it?”, Omega, 47, 1-16.

Schuschny, A. (2007): Método DEA y su aplicación al estudio del sector energético y las emisiones de CO2 en América Latina y el Caribe, CEPAL Serie estudios estadísticos prospectivos.

Sufian, F. (2011): "Benchmarking the efficiency of the Korean banking sector: a DEA approach”, Benchmarking: An International Journal, 18 (1), 107-127.

Tobin J. (1958): "Estimation of relationships for limited dependent variables”, Econometrica, 26, 24-36.

Tulkens, H. (1993): "On FDH efficiency analysis: Some methodological issues and applications to retail banking, courts, and urban transit”, Journal of Productivity Analysis, 4, (1), 183-210.

Veiderpass, A. y McKelvey, M. (2014): "Evaluating the performance of higher education institutions in Europe: A non-parametric efficiency analysis of 944 institutions”, Efficiency in Education. Workshop 19th - 20th September 2014 at The Work Foundation, London, U.K., 9. 
Vincová, K. (2005): “Using dea models to measure efficiency”, Technical University Košice, Biatec, XXIII, 8.

Voigt, S. y El Bialy, N. (2012): "Identifying the Determinants of Judicial Performance: Taxpayers'Money Well Spent?", Working Paper disponible en: http://papers.ssrn.com/sol3/papers.cfm?abstract_id=2241224.

Voigt, S. (2016): “Determinants of Judicial Efficiency: A Survey”, European Journal of Law and Economics, DOI 10.1007/s10657-016-9531-6. 